\title{
Second order rectifiability of varifolds of bounded mean curvature
}

\section{Mario Santilli ${ }^{1}$}

Received: 28 December 2020 / Accepted: 5 January 2021 / Published online: 12 April 2021

(c) The Author(s) 2021

\begin{abstract}
We prove that the support of an $m$ dimensional rectifiable varifold with a uniform lower bound on the density and bounded generalized mean curvature can be covered $\mathscr{H}^{m}$ almost everywhere by a countable union of $m$ dimensional submanifolds of class $\mathcal{C}^{2}$. The $\mathcal{C}^{2}$-regularity of the submanifolds is optimal.
\end{abstract}

Mathematics Subject Classification 49Q15 · 49Q20

\section{Introduction}

\section{Overview}

The concept of varifold goes back to the work of Almgren in the 60s and, since then, has played a central role in Geometric Measure Theory and in its applications. The definition is simple: an $m$-dimensional varifold $V$ in an open subset $\Omega$ of $\mathbf{R}^{n}$ is a Radon measure over $\Omega \times \mathbf{G}(n, m)$, where $\mathbf{G}(n, m)$ is the Grassmann manifold of all $m$ dimensional subspaces of $\mathbf{R}^{n}$. Three basic concepts can be associated to a varifold $V$ : the weight measure ${ }^{1}\|V\|$, the first variation ${ }^{2} \delta V$ and the total variation ${ }^{3}\|\delta V\|$. If the $m$ dimensional upper density $\boldsymbol{\Theta}^{* m}(\|V\|, x)$ is positive at $\|V\|$ a.e. $x \in \Omega$ and if $\|\delta V\|$ is a Radon measure over $\Omega$, then the rectifiability theorem of Allard $[1,5.5,2.8(5)]$ asserts that the set $\left\{x: 0<\Theta^{* m}(\|V\|, x)<\infty\right\}$ can be $\mathscr{H}^{m}$ almost covered by the union of a countable collection of $m$ dimensional submanifolds of class $\mathcal{C}^{1}$ of $\mathbf{R}^{n}$ and $\|V\|=\mathscr{H}^{m}\left\llcorner\boldsymbol{\Theta}^{m}(\|V\|, \cdot)\right.$.

\footnotetext{
${ }^{1}\|V\|$ is the Radon measure over $\Omega$ such that $\|V\|(U)=V(U \times \mathbf{G}(n, m))$ for each open subset $U$ of $\Omega$.

${ }^{2} \delta V(g)=\int \mathrm{D} g \bullet S_{\natural} d V(x, S)$ for every $g \in \mathcal{C}_{C}^{\infty}(\Omega)$, that is the initial rate of change of the total mass of the smooth deformation of $V$ with initial velocity given by $g$.

${ }^{3}\|\delta V\|$ is the largest Borel regular measure over $\Omega$ such that for each open set $U \subseteq \Omega$ the number $\|\delta V\|(U)$ equals $\sup \left\{\delta V(g): g \in \mathcal{C}_{c}^{\infty}\left(U, \mathbf{R}^{n}\right),|g| \leq 1\right\}$.
}

Communicated by A. Malchiodi.

$凶 \quad$ Mario Santilli

mario.santilli@math.uni-augsburg.de

1 Institut für Mathematik, Universität Augsburg, Universitätsstr. 14, 86159 Augsburg, Germany 
The regularity theorems of Allard and Duggan, [1,8] and [6, Theorem 2.1], allows to obtain the following statement: if $2 \leq m<p<\infty, \alpha<\infty$ and $V$ is an $m$ dimensional varifold such that $\boldsymbol{\Theta}^{m}(\|V\|, x) \geq 1$ for $\|V\|$ almost every $x \in \Omega$ and such that $\delta V(g) \leq$ $\alpha\left(\int|g|^{p /(p-1)} d\|V\|\right)^{(p-1) / p}$ for every $g \in \mathcal{C}_{c}^{\infty}\left(\Omega, \mathbf{R}^{n}\right)$, then a dense open subset of spt $\|V\|$ is an $m$ dimensional submanifold $M$ of class $W^{2, p}$. Notice that the integrability condition on the first variation in the previous sentence can be equivalently formulated requiring that the generalized mean curvature vector $\mathbf{h}(V, \cdot)$ of $V$ defined in [1,4.3] belongs to $L^{p}\left(\|V\|, \mathbf{R}^{n}\right)$. Assuming that there exists $\beta<2$ such that $\Theta^{m}(\|V\|, x) \leq \beta$ for $\|V\|$ almost every $x \in \Omega$, then the conclusion can be strengthened to $\|V\|(\Omega \sim M)=0$. However, without the latter hypothesis, the latter conclusion is known to be false. In fact Brakke constructed a varifold $V$ such that $\|\delta V\| \leq \alpha\|V\|$ (i.e. $\mathbf{h}(V, \cdot) \in L^{\infty}\left(\|V\|, \mathbf{R}^{n}\right)$ ) and $V$ cannot be locally represented as a graph of multiple-valued function around each point of a set of positive $\|V\|$ measure, see $[3,6.1]$. The Brakke's example essentially shows that the set of points with higher multiplicity of a varifold of bounded mean curvature might have an arbitrarily complicated structure.

On the other hand, considering that varifolds with locally bounded first variation provide the natural setting to study variational problems involving the mean curvature vector (henceforth they are the variational counterpart of $\mathcal{C}^{2}$ submanifolds), and also considering the aforementioned $W^{2, p}$-extension of the Allard regularity theory by Duggan, the following question naturally arises and plays a central role to understand fundamental aspects of varifolds geometry:

The $\mathcal{C}^{2}$ rectifiability problem. Let $V$ be an $m$ dimensional varifold in $\Omega$, whose total variation is a Radon measure and the $m$-dimensional density is uniformly lower bounded. Is it true that $\Omega$ can be covered, up to a set of $\|V\|$ measure zero, by a countable union of $m$ dimensional submanifolds of class $\mathcal{C}^{2} ?^{4}$

If $V$ is integral (i.e. the density of $\|V\|$ takes values in the set of positive integers) a positive full solution of the $\mathcal{C}^{2}$-rectifiability problem was given by Menne in [10] employing results from [9]. These two papers make essentially the final step in the line of research focused on the study of the tilt and height excess decay rates, which was initiated by the pioneering studies of Brakke in [3] and continued by Schätzle in the two papers $[19,20]$. The solution in [10] for the $\mathcal{C}^{2}$-rectifiability problem is purely analytical and employs the theory of second-order linear elliptic systems in combination with Almgren's theory of multiple-valued functions. Moreover the hypothesis of integrality plays a crucial role.

The main motivation of this paper arises from the idea that a purely differential geometric solution of the $\mathcal{C}^{2}$-rectifiability problem might exist, at least under certain additional (mild) assumptions on the first variation. Indeed this paper, in combination with [12,18], develops a new approach that allows to treat the $\mathcal{C}^{2}$ rectifiability problem in a purely differentialgeometric way. We exploit the non-variational nature of varifolds, developing a method that combines convex geometry and viscosity techniques (ABP method). The outcome is a purely geometric and significantly shorter solution of the $\mathcal{C}^{2}$ rectifiability problem, which does not require any integrality constrain but needs the first variation to be represented by a bounded function.

We state now the main result of this paper:

1.1 Theorem Suppose $1 \leq m<n$ are integers, $\Omega \subseteq \mathbf{R}^{n}$ is an open set, $V$ is an m dimensional varifold in $\Omega, S=\operatorname{spt}\|V\|$ and the following two conditions hold:

\footnotetext{
${ }^{4}$ We remark that the $\mathcal{C}^{2}$ regularity of the submanifolds is optimal, since there exist submanifolds of class $\mathcal{C}^{2}$ that cannot be almost covered by countably many submanifolds of class $\mathcal{C}^{2, \alpha}$ for every $\alpha>0$, see [2, Appendix]. A general study of the notion of higher-order rectifiability for sets has been initiated in [2] and a characterization in terms of higher order approximate derivatives has been obtained in [16].
} 
(1) there exists $0 \leq h<\infty$ such that $\|\delta V\| \leq h\|V\|$,

(2) there exists $\theta>0$ such that $\Theta^{m}(\|V\|, x) \geq \theta$ for $\|V\|$ a.e. $x \in \Omega$.

Then $S$ can be $\mathscr{H}^{m}$ almost covered by a countable collection of $m$ dimensional submanifolds of class $\mathcal{C}^{2}$ in $\mathbf{R}^{n}$.

The general geometric approach used to prove Theorem 1.1 promises to have several other applications. For example it has been recently used to obtain the characterization of the critical points of the anisotropic isoperimetric functional in [5].

\section{Method of the proof}

We explain here the key ideas of our proof.

For an arbitrary closed set $A \subseteq \mathbf{R}^{n}$ and an integer $m=0, \ldots, n$, we introduce the $m$-th stratum $A^{(m)}$ of $A$ to be the set of points where $A$ can be touched by balls from $n-m$ linearly independent directions (henceforth, $A^{(n)}$ is the set of points where no balls touch $A$ ). Here we mean that an open ball $B$ touches $A$ at a point $a \in A$ if $B \cap A=\varnothing$ and $a \in \operatorname{Clos}(B) \cap A$. It is proved in [12] that if $A$ is an arbitrary closed set then the $m$-th stratum $A^{(m)}$ can be always covered by countably many $m$ dimensional submanifolds of class $\mathcal{C}^{2}$. This structural property of arbitrary closed sets suggests a general strategy to prove $\mathcal{C}^{2}$-rectifiability, namely to check that $\mathscr{H}^{m}\left(A \sim A^{(m)}\right)=0$. We recall that simple examples show that the latter may fail to hold even for sets contained in countably many $m$ dimensional planes. Theorem 1.3 identifies a very general class of closed sets $A$ for which $\mathscr{H}^{m}\left(A \sim A^{(m)}\right)=0$ and the support of a varifold as in Theorem 1.1 belongs to this class.

In order to describe the class of closed sets addressed in Theorem 1.3, we need a few definitions. If $A \subseteq \mathbf{R}^{n}$ is closed, we define the generalized unit normal bundle of $A$ as

$$
N(A)=\left(A \times \mathbf{R}^{n}\right) \cap\left\{(a, u):|u|=1, \boldsymbol{\delta}_{A}(a+s u)=s \text { for some } s>0\right\}
$$

(here $\delta_{A}$ is the distance function from $A$ ). It is remarkable (and important for the method of this paper) that $N(A)$ is always a countably $n-1$ rectifiable subset of $\mathbf{R}^{n} \times \mathbf{R}^{n}$ (in the sense of $[8,3.2 .14])$. Notice that the $m$-th stratum can be expressed as

$$
A^{(m)}=A \cap\left\{a: 0<\mathscr{H}^{n-m-1}(N(A, a))<\infty\right\} \text { for } m=0, \ldots, n-1 .
$$

Roughly speaking the normal bundle $N(A)$ encodes the curvature properties of viscositytype of $A$. As for smooth varieties, these curvature properties can be conveniently described using a suitable real-valued symmetric bilinear form $Q_{A}(a, u)$, that can be defined at $\mathscr{H}^{n-1}$ almost every $(a, u) \in N(A)$. In analogy with the smooth case, we call it second fundamental form of $A$ at $a$ in the direction $u$.

What we have briefly described in the previous paragraph applies to all closed sets. We need now to identify a sufficiently general condition, expressed in terms of the normal bundle $N(A)$ and the $m$-th strata of $A$, which is also strong enough to allow to prove that $\mathscr{H}^{m}\left(A \sim A^{(m)}\right)=0$. This condition is expressed in the following definition.

1.2 Definition Suppose $A \subseteq \mathbf{R}^{n}$ is a closed set, $\Omega \subseteq \mathbf{R}^{n}$ is an open set and $1 \leq m<n$ is an integer. We say that $N(A)$ satisfies the $m$ dimensional Lusin $(N)$ condition in $\Omega$ if and only if the following property holds:

$$
\mathscr{H}^{n-1}(N(A) \cap\{(a, u): a \in Z\})=0
$$

for every $Z \subseteq A \cap \Omega$ with $\mathscr{H}^{m}\left(A^{(m)} \cap Z\right)=0$. 
Using the Lusin $(\mathrm{N})$ condition we can now formulate the following result, which is essentially the main result of the paper.

1.3 Theorem Suppose $1 \leq m<n$ are integers, $A \subseteq \mathbf{R}^{n}$ is a closed set and $\Omega \subseteq \mathbf{R}^{n}$ is an open set such that the following hypothesis are satisfied.

(I) $\mathscr{H}^{m}(A \cap K)<\infty$ whenever $K \subseteq \Omega$ is compact,

(II) $N(A)$ satisfies the $m$ dimensional Lusin $(N)$ condition in $\Omega$,

(III) for $\mathscr{H}^{m}$ a.e. $a \in A \cap \Omega$ there exists $v \in \mathbf{S}^{n-1}$ such that

$$
\lim _{r \rightarrow 0} r^{-1} \sup \{v \bullet(x-a): x \in \mathbf{B}(a, r) \cap A\}=0,
$$

(IV) there exists a non negative $\mathscr{H}^{n-1}$ measurable function $f$ on $N(A) \mid \Omega$ such that

$$
\begin{aligned}
& \operatorname{trace} Q_{A}(a, u) \leq f(a, u) \text { for } \mathscr{H}^{n-1} \text { a.e. }(a, u) \in N(A) \mid \Omega, \\
& \int_{K \cap A} \int_{\{z\} \times N(A, z)} f^{m} d \mathscr{H}^{n-m-1} d \mathscr{H}^{m} z<\infty,
\end{aligned}
$$

whenever $K \subseteq \Omega$ is compact.

Then $\mathscr{H}^{m}\left(A \cap \Omega \sim A^{(m)}\right)=0$. In particular, $A \cap \Omega$ is countably $\left(\mathscr{H}^{m}, m\right)$ rectifiable of class 2.

If $A$ is the closure in $\mathbf{R}^{n}$ of the support of a varifold $V$ as in Theorem 1.1, then the relation between the normal bundle $N(A)$, the second fundamental form $Q_{A}$, the Lusin $(\mathrm{N})$ condition and the generalized mean curvature $\mathbf{h}(V, \cdot)$ of $V$ has been clarified in $[18,3.8]$ : if $0 \leq h<\infty$ and $|\mathbf{h}(V, \cdot)| \leq h$ then $N(A)$ satisfies the $m$ dimensional Lusin $(\mathrm{N})$ condition in $\Omega$ and

$$
\text { trace } Q_{A}(a, u) \leq h \text { for } \mathscr{H}^{n-1} \text { a.e. }(a, u) \in N(A) \mid \Omega \text {. }
$$

This implies that the support of a varifold as in Theorem 1.1 fulfills the hypothesis of Theorem 1.3 .

To prove Theorem 1.3, firstly we use the Lusin $(\mathrm{N})$ condition to derive a Coarea-type formula in Theorem 3.7. Then we use such a formula in Lemma 3.10 to extend one of the key results of the theory of elliptic PDE's, the Alexandrov-Bakelmann-Pucci (ABP) estimate (see [4, Theorem 3.2]), to our geometric setting. We do not explicitly write such a formula in the statement of our results, since the study of the ABP inequality in the context of varifolds (or, more generally, in the abstract setting of closed sets) would be beyond the scope of the present paper; however, the reader might recognize the resemblance in inequality (20) of Lemma 3.10. The validity of the ABP inequality is the key to obtain Theorem 1.3. In fact, we use this inequality to generalize the techniques developed by Nadirashvili in [14] and Trudinger in [22, Theorem 1] to prove twice-differentiability almost everywhere of solutions of elliptic equations. A moment of reflection effectively reveals that $\mathscr{H}^{m}\left(A \sim A^{(m)}\right)=0$ is the natural counterpart in our geometric setting of the twice differentiability almost everywhere for functions.

\section{Further developments}

We conclude this section pointing out some possible future developments and limitations of our method in relation to the $\mathcal{C}^{2}$-rectifiability problem.

The next natural step would be to extend the approach of this paper to treat the $\mathcal{C}^{2}$ rectifiability problem for $m$ dimensional varifolds in $\mathbf{R}^{n}$ under the following more general integrability 
condition on the first variation (which appears to be the most general hypothesis under which the method of this paper might work, as explained at the end of this paragraph):

$\left(1^{\prime}\right)$ there exists $0 \leq \alpha<\infty$ such that $\left.\delta V(g) \leq \alpha\left(\int|g|^{m / m-1}\right) d\|V\|\right)^{m-1 / m}$ for every $g \in \mathcal{C}_{c}^{\infty}\left(\Omega, \mathbf{R}^{n}\right)$.

We notice that under the hypothesis $\left(1^{\prime}\right)$ the support of $\|V\|$ automatically satisfies (I) and (III) of Theorem 1.3, thanks to [1, 8.3] and [11, 11.3]. Therefore the main point would be to check condition (II) in Theorem 1.3. This is however a delicate point. In fact for varifolds of bounded mean curvature the Lusin $(\mathrm{N})$ condition is proved in [18] using the weak maximum principle in [23], which is not available for varifolds satisfying $\left(1^{\prime}\right)$. Therefore the aforementioned extension would require to investigate the validity of maximum principles for varifolds with unbounded mean curvature.

We finally remark that for varifolds satisfying a condition weaker than $\left(1^{\prime}\right)$ on the first variation (i.e. replace $m$ with some $1 \leq p<m$ in $\left(1^{\prime}\right)$ ), the differential-geometric approach of this paper seems to be not adequate. First of all, it is well known that in this case it may happen that the support of $\|V\|$ does not satisfy (I) of Theorem 1.3. More importantly, the ABP method naturally leads to consider the critical exponent $m$ for the integrability condition on the mean curvature in (IV) of Theorem 1.3. Therefore to treat this more general case the variational approach of Menne remains the only one in sight.

\section{Notation and preliminary results}

The open and closed balls of radius $r$ and center $a$ are respectively denoted by $\mathbf{U}(a, r)$ and $\mathbf{B}(a, r)$. The closure and the boundary in $\mathbf{R}^{n}$ of a set $A$ are denoted by Clos $A$ and $\partial A$. The symbol $\bullet$ denotes the standard inner product of $\mathbf{R}^{n}$. If $T$ is a linear subspace of $\mathbf{R}^{n}$, then $T_{\natural}: \mathbf{R}^{n} \rightarrow \mathbf{R}^{n}$ is the orthogonal projection onto $T$ and $T^{\perp}=\mathbf{R}^{n} \cap\{v: v \bullet u=0$ for $u \in T\}$. If $X$ and $Y$ are sets and $Z \subseteq X \times Y$ we set

$$
Z \mid S=Z \cap\{(x, y): x \in S\} \text { for } S \subseteq X
$$

The maps $\mathbf{p}, \mathbf{q}: \mathbf{R}^{n} \times \mathbf{R}^{n} \rightarrow \mathbf{R}^{n}$ are defined by $\mathbf{p}(x, v)=x$ and $\mathbf{q}(x, v)=v$.

We adopt the language of symmetric algebra to write in a compact form our formulas: if $f: V \rightarrow W$ is a linear map between vector spaces, then there exists a unique linear map $\bigodot_{2} f: \bigodot_{2} V \rightarrow \bigodot_{2} W$, which is the restriction of the unique unit-preserving algebra homeomorphism $\bigodot_{*} f: \bigodot_{*} V \rightarrow \bigodot_{*} W$ onto $\bigodot_{2} V$, see $[8,1.9]$. We adopt the terminology in $[8,3.2 .14]$ to treat rectifiable sets. Moreover we say that a set $A \subseteq \mathbf{R}^{n}$ is countably $\left(\mathscr{H}^{m}, m\right)$ rectifiable of class 2 if $A$ can be $\mathscr{H}^{m}$ almost covered by the union of countably many $m$ dimensional submanifolds of class 2 of $\mathbf{R}^{n}$.

\subsection{Curvatures of arbitrary closed sets}

The reference for this section is [17].

Suppose $A$ is a closed subset of $\mathbf{R}^{n}$. The distance function to $A$ is denoted by $\boldsymbol{\delta}_{A}$. If $U$ is the set of all $x \in \mathbf{R}^{n}$ such that there exists a unique $a \in A$ with $|x-a|=\delta_{A}(x)$, we define the nearest point projection onto $A$ as the map $\xi_{A}$ characterised by the requirement

$$
\left|x-\xi_{A}(x)\right|=\delta_{A}(x) \text { for } x \in U .
$$


Let $U(A)=\operatorname{dmn} \xi_{A} \sim A$. The functions $\boldsymbol{v}_{A}$ and $\psi_{A}$ are defined by

$$
\boldsymbol{v}_{A}(z)=\boldsymbol{\delta}_{A}(z)^{-1}\left(z-\boldsymbol{\xi}_{A}(z)\right) \text { and } \boldsymbol{\psi}_{A}(z)=\left(\boldsymbol{\xi}_{A}(z), \boldsymbol{v}_{A}(z)\right) \text {, }
$$

whenever $z \in U(A)$. We define (see $[17,3.6,3.7]$ ) the upper semicontinuous function $\rho(A, \cdot)$ setting

$$
\rho(A, x)=\sup \left\{t: \boldsymbol{\delta}_{A}\left(\boldsymbol{\xi}_{A}(x)+t\left(x-\boldsymbol{\xi}_{A}(x)\right)\right)=t \boldsymbol{\delta}_{A}(x)\right\} \text { for } x \in U(A),
$$

and we say that $x \in U(A)$ is a regular point of $\boldsymbol{\xi}_{A}$ if and only if $\boldsymbol{\xi}_{A}$ is approximately differentiable 5 at $x$ with symmetric approximate differential and ap $\lim _{y \rightarrow x} \rho(A, y) \geq \rho(A, x)>1$. The set of regular points of $\xi_{A}$ is denoted by $R(A)$. It is proved in [17, 3.14] that $\mathscr{L}^{n}\left(\mathbf{R}^{n} \sim(A \cup R(A))\right)=0$ and if $x \in R(A)$ then $\xi_{A}(x)+t\left(x-\xi_{A}(x)\right) \in R(A)$ for every $0<t<\rho(A, x)$. Moreover, $\boldsymbol{\psi}_{A} \mid\left\{x: \boldsymbol{\delta}_{A}(x)=r, \rho(A, x) \geq \lambda\right\}$ is a bi-lipschitzian homeomorphism whenever $r>0$ and $\lambda>1$, see [17, 3.17(1)].

Combining these two facts, we now briefly describe how a general notion of second fundamental form for arbitrary closed sets has been introduced in [17, section 4]. This notion will be repeatedly used in the rest of this paper. First of all, we define the generalized unit normal bundle of $A$ as

$$
N(A)=\left(A \times \mathbf{S}^{n-1}\right) \cap\left\{(a, u): \boldsymbol{\delta}_{A}(a+s u)=s \text { for some } s>0\right\},
$$

with $N(A, a)=\{v:(a, v) \in N(A)\}$ for $a \in A$. Since

$$
N(A)=\bigcup_{r>0} \psi_{A}\left[\left\{x: \delta_{A}(x)=r, \rho(A, x) \geq \lambda\right\}\right] \text { for every } \lambda>1,
$$

one uses the rectifiability properties of the distance sets $\left\{x: \boldsymbol{\delta}_{A}(x)=r\right\}$ (see [17, 2.13]) to conclude that $N(A)$ is a countably $n-1$ rectifiable subset of $\mathbf{R}^{n} \times \mathbf{S}^{n-1}$. Then we introduce the following definition: if $x \in R(A)$ then we say that $\psi_{A}(x)$ is a regular point of $N(A)$, and we denote the set of all regular points of $N(A)$ by $R(N(A))$. One may check (see [17, 4.5]) that $\mathscr{H}^{n-1}[N(A) \sim R(N(A))]=0$. For every $(a, u) \in R(N(A))$, if $x \in R(A)$ and $\psi_{A}(x)=(a, u)$, we define

$$
T_{A}(a, u)=\operatorname{im} \operatorname{apD} \xi_{A}(x),
$$

and we define a symmetric bilinear form $Q(a, u): T_{A}(a, u) \times T_{A}(a, u) \rightarrow \mathbf{R}$ which maps $\left(\tau, \tau_{1}\right) \in T_{A}(a, u) \times T_{A}(a, u)$ into

$$
Q_{A}(a, u)\left(\tau, \tau_{1}\right)=\tau \bullet \operatorname{apD} v_{A}(x)\left(\sigma_{1}\right) ;
$$

here $\sigma_{1} \in \mathbf{R}^{n}$ is any vector such that ap $\mathrm{D} \boldsymbol{\xi}_{A}(x)\left(\sigma_{1}\right)=\tau_{1}$. This is a well-posed definition, see $[17,4.6,4.8]$. We call $Q_{A}(a, u)$ second fundamental form of $A$ at a in the directionu. It is not difficult to check that if $A$ is smooth submanifold, then $Q_{A}$ agrees with the classical notion of differential geometry. Moreover, if $(a, u) \in R(N(A))$ we define the principal curvatures of $A$ at $(a, u)$ to be the numbers

$$
\kappa_{A, 1}(a, u) \leq \cdots \leq \kappa_{A, n-1}(a, u)
$$

such that $\kappa_{A, m+1}(a, u)=\infty, \kappa_{A, 1}(a, u), \ldots, \kappa_{A, m}(a, u)$ are the eigenvalues of $Q_{A}(a, u)$ and $m=\operatorname{dim} T_{A}(a, u)$.

$\overline{5}$ See $[17,2.4,2.6]$ for the definition of approximate differentiability. 


\subsection{The $\mathcal{C}^{2}$-rectifiable stratification}

The reference for this section is [12].

Suppose $A \subseteq \mathbf{R}^{n}$ is a closed subset of $\mathbf{R}^{n}$. For each $a \in A$ we define (see [12, 4.1, 4.2]) the closed convex subset

$$
\operatorname{Dis}(A, a)=\left\{v:|v|=\boldsymbol{\delta}_{A}(a+v)\right\}
$$

and we notice that $N(A, a)=\{v /|v|: 0 \neq v \in \operatorname{Dis}(A, a)\}$. For every integer $0 \leq m \leq n$ we define the $m$-th stratum of $A$ by

$$
A^{(m)}=A \cap\{a: \operatorname{dim} \operatorname{Dis}(A, a)=n-m\} ;
$$

this is a Borel set which is countably $m$ rectifiable and countably $\left(\mathscr{H}^{m}, m\right)$ rectifiable of class 2 ; see [12, 4.12]. This definition agrees with [17, 5.1] by [12,4.4]. Moreover, one may use the classical Coarea formula for functions to infer that

$$
\begin{aligned}
A^{(m)} & =A \cap\left\{a: 0<\mathscr{H}^{n-m-1}(N(A, a))<\infty\right\} \text { if } m=0, \ldots, n-1, \\
A^{(n)} & =A \cap\{a: N(A, a)=\varnothing\} .
\end{aligned}
$$

\subsection{Curvature under diffeomorphic deformations}

In this section we prove an explicit formula for the second fundamental form $Q_{F[A]}$ of a diffeomorphic deformation $F[A]$ of an arbitrary closed set $A$, in terms of $Q_{A}$. This formula appears to be new even in the smooth setting.

2.1 Lemma Suppose $A \subseteq \mathbf{R}^{n}$ is a closed set, $F: \mathbf{R}^{n} \rightarrow \mathbf{R}^{n}$ is a diffeomorphism of class 2 onto $\mathbf{R}^{n}$ and $v_{F}: \mathbf{R}^{n} \times \mathbf{S}^{n-1} \rightarrow \mathbf{R}^{n} \times \mathbf{S}^{n-1}$ is given by

$$
v_{F}(a, u)=\left(F(a), \frac{\left(\mathrm{D} F(a)^{-1}\right)^{*}(u)}{\left|\left(\mathrm{D} F(a)^{-1}\right)^{*}(u)\right|}\right) \quad \text { whenever }(a, u) \in \mathbf{R}^{n} \times \mathbf{S}^{n-1} .
$$

Then $v_{F}$ is a diffeomorphism of class 1 onto $\mathbf{R}^{n} \times \mathbf{S}^{n-1},\left(v_{F}\right)^{-1}=v_{F^{-1}}$ and

$$
v_{F}(N(A))=N(F(A)) \text {. }
$$

In particular, $F\left(A^{(m)}\right)=F(A)^{(m)}$ for $m=0, \ldots, n$.

Proof A direct computation shows that $v_{F}$ is a diffeomorphism of class 1 onto $\mathbf{R}^{n} \times \mathbf{S}^{n-1}$ with $\left(v_{F}\right)^{-1}=v_{F^{-1}}$.

If $(a, u) \in N(A)$ and $r>0$ such that $\mathbf{U}(a+r u, r) \cap A=\varnothing$, we let

$$
v=\left(\mathrm{D} F(a)^{-1}\right)^{*}(u), \quad W=F(\mathbf{U}(a+r u, r)), \quad S=\partial W .
$$

Since $S=F(\partial \mathbf{U}(a+r u, r))$, by $[8,3.1 .21]$ we conclude that

$$
\mathrm{D} F(a)(\operatorname{Tan}(\partial \mathbf{U}(a+r u, r), a))=\operatorname{Tan}(S, F(a)),
$$

and, consequently, $v \in \operatorname{Nor}(S, F(a))$. If $s=\operatorname{reach}(S, F(a))$ (see [7, 4.1]), then by [7, 4.11, 4.8(12)] we conclude that $s>0$,

$$
\mathbf{U}(F(a)+s(v /|v|), s) \cap S=\varnothing
$$

and we deduce that

$$
\text { either } \mathbf{U}(F(a)+s(v /|v|), s) \subseteq W \text { or } \mathbf{U}(F(a)+s(v /|v|), s) \subseteq \mathbf{R}^{n} \sim \operatorname{Clos} W .
$$


If $\gamma(t)=F(a+t u)$ for $t \in \mathbf{R}$, noting that $\dot{\gamma}(0) \bullet v=1$ and

$$
\mathrm{D}_{t}(|\gamma(t)-F(a)-s(v /|v|)|)(0)=-1 /|v|,
$$

we conclude that $\gamma(t) \in \mathbf{U}(F(a)+s(v /|v|), s)$ for $t>0$ sufficiently small,

$$
\mathbf{U}(F(a)+s(v /|v|), s) \subseteq W \quad \text { and } \quad v_{F}(a, u) \in N(F(A)) .
$$

Therefore $v_{F}(N(A)) \subseteq N(F(A))$ and replacing $F$ by $F^{-1}$ and $A$ by $F(A)$ we conclude

$$
v_{F}(N(A))=N(F(A)) .
$$

Noting that for each $a \in \mathbf{R}^{n}$ the function mapping $u \in \mathbf{S}^{n-1}$ onto $\mathbf{q}\left(v_{F}(a, u)\right)$ is a diffeomorphism onto $\mathbf{S}^{n-1}$ the postscript follows from (1) and (2).

2.2 Theorem Suppose A is a closed subset of $\mathbf{R}^{n}$ and $F: \mathbf{R}^{n} \rightarrow \mathbf{R}^{n}$ is a diffeomorphism of class 2 onto $\mathbf{R}^{n}$.

Then ( see Lemma 2.1) D $F(a)\left(T_{A}(a, u)\right)=T_{F(A)}\left(v_{F}(a, u)\right)$ and

$$
\begin{aligned}
& Q_{F(A)}\left(v_{F}(a, u)\right) \circ \bigodot_{2}\left(\mathrm{D} F(a) \mid T_{A}(a, u)\right) \\
& \quad=\left|\left(\mathrm{D} F(a)^{-1}\right)^{*}(u)\right|^{-1} Q_{A}(a, u) \\
& \quad+\left(\mathrm{D}^{2} F(a) \mid \bigodot_{2} T_{A}(a, u)\right) \bullet\left(\left(\mathrm{D} F(a)^{-1}\right)^{*}(u) /\left|\left(\mathrm{D} F(a)^{-1}\right)^{*}(u)\right|\right),
\end{aligned}
$$

for $\mathscr{H}^{n-1}$ a.e. $(a, u) \in N(A)$.

Proof We define $g: \mathbf{R}^{n} \times \mathbf{R}^{n} \rightarrow \mathbf{R}^{n}$ to be

$$
g(a, u)=\left(\mathrm{D} F(a)^{-1}\right)^{*}(u) \text { for }(a, u) \in \mathbf{R}^{n} \times \mathbf{R}^{n} .
$$

To compute $\mathrm{D} g$, we first notice that

$$
g=\left[\beta \circ\left(\alpha \times \mathbf{1}_{\mathbf{R}^{n}}\right) \circ\left(\iota \times \mathbf{1}_{\mathbf{R}^{n}}\right) \circ\left(\mathrm{D} F \times \mathbf{1}_{\mathbf{R}^{n}}\right)\right],
$$

where $\iota(T)=T^{-1}$ for every isomorphism $T$ of $\mathbf{R}^{n}, \alpha(T)=T^{*}$ for $T \in \operatorname{Hom}\left(\mathbf{R}^{n}, \mathbf{R}^{n}\right)$ and $\beta(T, u)=T(u)$ for every $(T, u) \in \operatorname{Hom}\left(\mathbf{R}^{n}, \mathbf{R}^{n}\right) \times \mathbf{R}^{n}$. Then differentiating such a composition of maps one obtains that

$$
\begin{aligned}
\mathrm{D} g(a, u)(\tau, \sigma)=\left(\mathrm{D} F(a)^{-1}\right)^{*}(\sigma) & \\
& +\left[\left(\mathrm{D} F(a)^{-1}\right)^{*} \circ \mathrm{D}(\mathrm{D} F)(a)(\tau)^{*} \circ\left(\mathrm{D} F(a)^{-1}\right)^{*}\right](u),
\end{aligned}
$$

for $(a, u)$ and $(\tau, \sigma)$ in $\mathbf{R}^{n} \times \mathbf{R}^{n}$. Moreover, one can easily compute that

$$
\mathrm{D}\left(\frac{g}{|g|}\right)(a, u)=\frac{1}{|g(a, u)|}\left[\mathrm{D} g(a, u)-\left(\mathrm{D} g(a, u) \bullet \frac{g(a, u)}{|g(a, u)|}\right) \frac{g(a, u)}{|g(a, u)|}\right]
$$

for $(a, u) \in \mathbf{R}^{n} \times\left(\mathbf{R}^{n} \sim\{0\}\right)$ and $(\tau, \sigma) \in \mathbf{R}^{n} \times \mathbf{R}^{n}$.

Let $\theta$ be $\mathscr{H}^{n-1}\left\llcorner N(A)\right.$ measurable and $\mathscr{H}^{n-1}\llcorner N(A)$ almost positive function such that $\theta \mathscr{H}^{n-1}\llcorner N(A)$ is a Radon measure. Noting Lemma 2.1, we define

$$
\mu=\left(\theta \circ v_{F^{-1}}\right) \mathscr{H}^{n-1}\llcorner N(F[A])
$$

and we apply $[17, \mathrm{~B} .1]$ with $\gamma=\operatorname{Lip}\left(v_{F} \mid v_{F^{-1}}[K]\right)$ to conclude that

$$
\mu(K) \leq \gamma^{n-1} \int_{N(A) \cap v_{F^{-1}}[K]} \theta d \mathscr{H}^{n-1}<\infty
$$


whenever $K \subseteq \mathbf{R}^{n} \times \mathbf{S}^{n-1}$ is compact. Let $\psi=\theta \mathscr{H}^{n-1}\llcorner N(A)$. Noting again Lemma 2.1, one may use $[17,4.11(1)]$ and $\left[17\right.$, B.2] to see that for $\mathscr{H}^{n-1}$ a.e. $(a, u) \in N(A)$ the approximate tangent cones $\operatorname{Tan}^{n-1}(\psi,(a, u))$ and $\operatorname{Tan}^{n-1}\left(\mu, v_{F}(a, u)\right)$ are $n-1$ dimensional planes in $\mathbf{R}^{n} \times \mathbf{R}^{n}$ and

$$
\mathrm{D} v_{F}(a, u)\left[\operatorname{Tan}^{n-1}(\psi,(a, u))\right]=\operatorname{Tan}^{n-1}\left(\mu, v_{F}(a, u)\right) .
$$

Employing [17, 4.11(2)] one infers for $\mathscr{H}^{n-1}$ a.e. $(a, u) \in N(A)$ that

$$
\begin{aligned}
& \mathrm{D} F(a)\left[T_{A}(a, u)\right]=T_{F[A]}\left(v_{F}(a, u)\right), \\
& Q_{F[A]}\left(v_{F}(a, u)\right)\left(\mathrm{D} F(a)(\tau), \mathrm{D} F(a)\left(\tau_{1}\right)\right) \\
& \quad=\mathrm{D} F(a)(\tau) \bullet \mathrm{D}(g /|g|)(a, u)\left(\tau_{1}, \sigma_{1}\right)
\end{aligned}
$$

whenever $\tau \in T_{A}(a, u), \tau_{1} \in T_{A}(a, u)$ and $\left(\tau_{1}, \sigma_{1}\right) \in \operatorname{Tan}^{n-1}(\psi,(a, u))$. Since

$$
T_{A}(a, u) \subseteq\{v: v \bullet u=0\}
$$

for $\mathscr{H}^{n-1}$ a.e. $(a, u) \in N(A)$ by $[17,4.5,4.8]$, it follows from (5) that

$$
\mathrm{D} F(a)(\tau) \bullet(g /|g|)(a, u)=|g(a, u)|^{-1} u \bullet \tau=0
$$

for $\mathscr{H}^{n-1}$ a.e. $(a, u) \in N(A)$ and for every $\tau \in T_{A}(a, u)$. Therefore combining (6), (4), (7) and (3) we obtain for $\mathscr{H}^{n-1}$ a.e. $(a, u) \in N(A)$ that

$$
\begin{aligned}
& Q_{F[A]}\left(v_{F}(a, u)\right)\left(\mathrm{D} F(a)(\tau), \mathrm{D} F(a)\left(\tau_{1}\right)\right) \\
&=|g(a, u)|^{-1} \mathrm{D} F(a)(\tau) \bullet \mathrm{D} g(a, u)\left(\tau_{1}, \sigma_{1}\right) \\
&=|g(a, u)|^{-1}\left[\left(\tau \bullet \sigma_{1}\right)+\mathrm{D}(\mathrm{D} F)(a)\left(\tau_{1}\right)(\tau) \bullet\left(\mathrm{D} F(a)^{-1}\right)^{*}(u)\right]
\end{aligned}
$$

for every $\tau, \tau_{1} \in T_{A}(a, u)$ and $\left(\tau_{1}, \sigma_{1}\right) \in \operatorname{Tan}^{n-1}(\psi,(a, u))$. This is our conclusion by [17, 4.11(2)].

\section{A sufficient condition for $\mathcal{C}^{2}$-rectifiability for closed sets}

This section is the main technical part of the paper. We work in the abstract setting of closed subsets whose generalized unit normal bundle satisfies the Lusin (N) condition. The main point here is to provide a general criterion for $\mathcal{C}^{2}$-rectifiability (see Theorem 1.3). Then, in the next section we verify that the support of a varifold as in Theorem 1.1 satisfies the hypothesis of this criterion, thus obtaining the announced result for varifolds.

3.1 Definition Suppose $A \subseteq \mathbf{R}^{n}$ is a closed set, $\Omega \subseteq \mathbf{R}^{n}$ is an open set and $1 \leq m<n$ is an integer. We say that $N(A)$ satisfies the $m$ dimensional Lusin $(N)$ condition in $\Omega$ if and only if

$$
\mathscr{H}^{n-1}(N(A) \mid S)=0, \quad \text { whenever } S \subseteq A \cap \Omega \text { such that } \mathscr{H}^{m}\left(A^{(m)} \cap S\right)=0 \text {. }
$$

In case $\Omega=\mathbf{R}^{n}$, we say that $N(A)$ satisfies the $m$ dimensional Lusin $(N)$ condition.

We have introduced this terminology in analogy with the theory of functions: $f: \mathbf{R}^{n} \rightarrow \mathbf{R}^{n}$ is said to satisfy the Lusin (N) condition if $\mathscr{L}^{n}(f(A))=0$ whenever $\mathscr{L}^{n}(A)=0$, see [13]. 
3.2 Remark Suppose $A$ is a closed subset of $\mathbf{R}^{n}, \Omega$ is an open subset of $\mathbf{R}^{n}$ and $C=$ $\operatorname{Clos}(A \cap \Omega)$. Then one may easily check that

$$
N(A)|\Omega=N(C)| \Omega .
$$

It follows that $A^{(m)} \cap \Omega=C^{(m)} \cap \Omega$ for every $m=0, \ldots, n$ by (1), whence we deduce that if $N(A)$ satisfies the $m$ dimensional Lusin $(N)$ condition in $\Omega$, then $N(C)$ satisfies the $m$ dimensional Lusin $(N)$ condition in $\Omega$. Moreover,

$$
Q_{A}(\zeta)=Q_{C}(\zeta)
$$

for $\mathscr{H}^{n-1}$ a.e. $\zeta \in N(A) \mid \Omega$ by $[17,4.14]$.

3.3 Remark If $N(A)$ satisfies the $m$ dimensional Lusin $(\mathrm{N})$ condition in $\Omega$ then it follows from $[17,6.1]$ and $[12,4.12]$ that

$$
\operatorname{dim} T_{A}(a, u)=m \text { for } \mathscr{H}^{n-1} \text { a.e. }(a, u) \in N(A) \mid \Omega .
$$

3.4 Lemma Suppose $U \subseteq \mathbf{R}^{n}$ is open, $A \subseteq \mathbf{R}^{n}$ is closed, $N(A)$ satisfies the $m$ dimensional Lusin $(N)$ condition in $U$ and $F: \mathbf{R}^{n} \rightarrow \mathbf{R}^{n}$ is a diffeomorphism of class 2 onto $\mathbf{R}^{n}$.

Then $N(F(A))$ satisfies the $m$ dimensional Lusin $(N)$ condition in $F(U)$.

Proof Suppose $S \subseteq F(A) \cap F(U)$ such that $\mathscr{H}^{m}\left(F(A)^{(m)} \cap S\right)=0$. Since $F^{-1}(S) \subseteq A \cap U$ and $0=\mathscr{H}^{m}\left(F^{-1}\left(S \cap F(A)^{(m)}\right)\right)=\mathscr{H}^{m}\left(F^{-1}(S) \cap A^{(m)}\right)$ by Lemma 2.1, it follows by the Lusin $(\mathrm{N})$ condition of $N(A)$ that

$$
\mathscr{H}^{n-1}\left(N(A) \mid F^{-1}(S)\right)=0 .
$$

Then Lemma 2.1 implies that

$$
\nu_{F}\left(N(A) \mid F^{-1}(S)\right)=N(F(A)) \mid S, \quad \mathscr{H}^{n-1}(N(F(A)) \mid S)=0 .
$$

The preservation of the Lusin $(\mathrm{N})$ condition under diffeomorphisms is a subtle point. In fact, the following example shows that if we had define the Lusin condition in Definition 3.1 replacing $\mathscr{H}^{n-1}(N(A) \mid S)=0$ with the weaker property $\mathscr{H}^{n-1}(\mathbf{q}(N(A) \mid S))=0$, then the resulting condition would not be preserved under diffeomorphisms, as the following example shows for $n=3$ and $m=2$.

3.5 Example Suppose $A=\mathbf{R}^{3} \cap\{(x, y, z): z=|x|\}$ and $F: \mathbf{R}^{3} \rightarrow \mathbf{R}^{3}$ is given by $F(x, y, z)=\left(x, y, z+1-x^{2}-y^{2}\right)$ for $(x, y, z) \in \mathbf{R}^{3}$. Then one readily verifies that $\mathscr{H}^{2}(\mathbf{q}(N(A)))=0$. On the other hand,

$$
\begin{array}{r}
A^{(1)}=\mathbf{R}^{3} \cap\{(x, y, z): x=z=0\}, \\
F(A)^{(1)}=F\left(A^{(1)}\right)=\left\{\left(0, y, 1-y^{2}\right): y \in \mathbf{R}\right\},
\end{array}
$$

and the relative interior in $\mathbf{S}^{2}$ of $\mathbf{q}\left[N(F(A)) \mid F(A)^{(1)}\right]$ is non empty, as one may see by computing explicitly $F[A]$.

3.6 Remark The Lusin $(\mathrm{N})$ condition has been recently obtained in [5] also for varifolds of codimension 1 whose anisotropic first variation with respect to a uniformly convex integrand is represented by a bounded function, and plays a central role in the characterization of the critical points of the anisotropic isoperimetric functional. 
One of the main consequences of the Lusin $(\mathrm{N})$ condition is the following Coarea-type formula, whose proof is given in $[18,3.3]$.

3.7 Theorem Suppose $1 \leq m<n$ is an integer, $\Omega \subseteq \mathbf{R}^{n}$ is open, $A \subseteq \mathbf{R}^{n}$ is closed and $N(A)$ satisfies the $m$ dimensional Lusin $(N)$ condition in $\Omega$.

Then for every $\mathscr{H}^{n-1}$ measurable set $B \subseteq N(A) \mid \Omega$,

$$
\begin{aligned}
& \int_{\mathbf{S}^{n-1}} \mathscr{H}^{0}\{a:(a, u) \in B\} d \mathscr{H}^{n-1} u \\
& \quad=\int_{A} \int_{\{z\} \times\{\eta:(z, \eta) \in B\}}\left|\operatorname{discr} Q_{A}\right| d \mathscr{H}^{n-m-1} d \mathscr{H}^{m} z .
\end{aligned}
$$

We need the following simple fact from linear algebra in the proof of the next result.

3.8 Lemma Suppose $V$ and $W$ are finite dimensional vector spaces with inner products such that $\operatorname{dim} V=m$ and $\operatorname{dim} W=n, f \in \operatorname{Hom}(V, W), 0<t<\infty$ and $b \in \bigodot^{2} W$ such that $b(w, w) \leq t|w|^{2}$ whenever $w \in W$.

Then

$$
\|f\|^{2} \operatorname{trace}(b)+(1-n) t\|f\|^{2} \leq \operatorname{trace}\left(b \circ \bigodot_{2} f\right) \leq m t\|f\|^{2} .
$$

Proof By [8, 1.7.3] we can choose an orthonormal basis $v_{1}, \ldots, v_{m}$ of $V$ and an orthonormal basis $w_{1}, \ldots, w_{n}$ of $W$ such that

$$
\left(f^{*} \circ f\right)\left(v_{i}\right) \bullet v_{j}=0 \text { and } b\left(w_{i}, w_{j}\right)=0,
$$

whenever $i \neq j$. If we define $c(w, z)=t(w \bullet z)-b(w, z)$ whenever $w, z \in W$, noting $\|f\|=\left\|f^{*}\right\|$ by $[8,1.7 .6]$, we compute

$$
\begin{aligned}
& \operatorname{trace}\left(c \circ \bigodot_{2} f\right)=\sum_{i=1}^{m} \sum_{j=1}^{n}\left(f\left(v_{i}\right) \bullet w_{j}\right)^{2} c\left(w_{j}, w_{j}\right) \\
& \quad=\sum_{j=1}^{n}\left|f^{*}\left(w_{j}\right)\right|^{2} c\left(w_{j}, w_{j}\right) \leq\|f\|^{2}(n t-\operatorname{trace} b), \\
& \quad \operatorname{trace}\left(c \circ \bigodot_{2} f\right)=t \sum_{i=1}^{m}\left|f\left(v_{i}\right)\right|^{2}-\operatorname{trace}\left(b \circ \bigodot_{2} f\right) \geq t\|f\|^{2}-\operatorname{trace}\left(b \circ \bigodot_{2} f\right) .
\end{aligned}
$$

Combining the two equations we get the left side. The right side is trivial.

3.9 Definition If $0<t<\infty, a \in \mathbf{R}^{n}$ an $T \in \mathbf{G}(n, n-1)$, we define

$$
C_{t}(T, a)=\mathbf{R}^{n} \cap\left\{x:\left|T_{\natural}(x-a)\right|<t,\left|T_{\natural}^{\perp}(x-a)\right|<t\right\} .
$$

The criterion for second-order-differentiability in Theorem 1.3, that is the central result of this section, can be deduced by standard arguments from the somewhat more subtle result in Lemma 3.10.

3.10 Lemma (Main Lemma) If $1 \leq m<n$ are integers, then there exist $0<\delta<\infty$ and $0<\sigma<\infty$ such that the following statement holds.

If $A \subseteq \mathbf{R}^{n}$ is a closed set, $a \in A, 0<r<\infty, T \in \mathbf{G}(n, n-1)$ and the following three conditions hold,

(I) $N(A)$ satisfies the $m$ dimensional Lusin $(N)$ condition in $C_{4 r}(T, a)$,

(II) there exists $v \in \mathbf{S}^{n-1}$ such that $T_{\natural}(v)=0$ and

$$
\sup \left\{v \bullet(x-a): x \in \operatorname{Clos}\left(A \cap C_{4 r}(T, a)\right)\right\} \leq r / 16,
$$


(III) there exists a nonnegative $\mathscr{H}^{n-1}$ measurable function $f$ on $N(A)$ such that

$$
\begin{aligned}
& \operatorname{trace} Q_{A}(x, u) \leq f(x, u) \text { for } \mathscr{H}^{n-1} \text { a.e. }(x, u) \in N(A) \mid C_{2 r}(T, a), \\
& \int_{C_{2 r}(T, a) \cap A} \int_{\{z\} \times N(A, z)} f^{m} d \mathscr{H}^{n-m-1} d \mathscr{H}^{m} z \leq \delta,
\end{aligned}
$$

then there exists a Borel set $M \subseteq N(A) \mid \mathrm{Clos} C_{(11 / 8) r}(T, a)$ such that

$$
\begin{aligned}
& \mathscr{H}^{m}(\mathbf{p}[M]) \geq \sigma r^{m}, \\
& \boldsymbol{\delta}_{A}(x+(r / 2) u)=r / 2 \quad \text { if }(x, u) \in M, \\
& \mathscr{H}^{n-m-1}\{v:(x, v) \in M\}>0 \text { if } x \in \mathbf{p}[M], \\
& \mathbf{p}[M] \subseteq A^{(m)} .
\end{aligned}
$$

Proof We assume $a=0$ and we let $C_{t}=C_{t}(T, 0)$ whenever $0<t<\infty$.

By Remark 3.2 we notice that $N\left(\operatorname{Clos}\left(A \cap C_{4 r}\right)\right)$ satisfies the $m$ dimensional Lusin $(\mathrm{N})$ condition in $C_{4 r}$ and we replace $A$ with $\operatorname{Clos}\left(A \cap C_{4 r}\right)$. We consider the diffeomorphism $F: \mathbf{R}^{n} \rightarrow \mathbf{R}^{n}$ given by

$$
F(x)=x+(r / 8) v-(4 r)^{-1}\left|T_{\natural}(x)\right|^{2} v \quad \text { for } x \in \mathbf{R}^{n}
$$

and we compute

$$
\begin{aligned}
F^{-1}(x) & =x-(r / 8) v+(4 r)^{-1}\left|T_{\natural}(x)\right|^{2} v, \\
\mathrm{D} F(x)(u) & =u-(2 r)^{-1}\left(T_{\natural}(x) \bullet T_{\natural}(u)\right) v, \\
\mathrm{D} F^{-1}(x)(u) & =u+(2 r)^{-1}\left(T_{\natural}(x) \bullet T_{\natural}(u)\right) v, \\
\mathrm{D}^{2} F^{-1}(x)\left(u_{1}, u_{2}\right) & =(2 r)^{-1}\left(T_{\natural}\left(u_{1}\right) \bullet T_{\natural}\left(u_{2}\right)\right) v,
\end{aligned}
$$

for $x, u, u_{1}, u_{2} \in \mathbf{R}^{n}$. Moreover we notice

$$
\begin{aligned}
& F^{-1}\left[\operatorname{Clos} C_{r}\right] \subseteq \operatorname{Clos} C_{(11 / 8) r}, \\
& \sup _{x \in A} F(x) \bullet v \leq 3 r / 16, \quad \sup _{x \in A,\left|T_{\natural}(x)\right| \geq r} F(x) \bullet v \leq-r / 16 .
\end{aligned}
$$

Suppose $L$ is the set of $(z, \eta) \in\left(F[A] \cap C \operatorname{los} C_{r}\right) \times \mathbf{S}^{n-1}$ such that $(w-z) \bullet \eta \leq 0$ whenever $w \in F[A]$. We observe that $L$ is compact and, noting (8),

$$
L \subseteq N(F[A]) \mid F\left[\operatorname{Clos} C_{(11 / 8) r}\right] .
$$

We define $v_{F^{-1}}$ as in Lemma 2.1 and we prove that

$$
\boldsymbol{\delta}_{A}\left(F^{-1}(z)+(r / 2)\left(\mathbf{q} \circ v_{F^{-1}}\right)(z, \eta)\right)=r / 2 \text { for }(z, \eta) \in L .
$$

In fact, if $x=F^{-1}(z), \zeta=\left(\mathbf{q} \circ v_{F^{-1}}\right)(z, \eta)$ and $y \in A$, we compute

$$
\begin{aligned}
\left|\mathrm{D} F(x)^{*}(\eta)\right|^{-1} \eta= & \left(\mathrm{D} F(x)^{*}\right)^{-1}(\zeta)=\zeta+(2 r)^{-1}(v \bullet \zeta) T_{\natural}(x), \\
0 \geq & \left(D F(x)^{*}\right)^{-1}(\zeta) \bullet(F(y)-F(x)) \\
= & \zeta \bullet(y-x)+(4 r)^{-1}\left(\left|T_{\natural}(x)\right|^{2}-\left|T_{\natural}(y)\right|^{2}\right)(v \bullet \zeta) \\
& +(2 r)^{-1}\left(T_{\natural}(x) \bullet(y-x)\right)(v \bullet \zeta) \\
= & \zeta \bullet(y-x)-(4 r)^{-1}\left|T_{\natural}(y-x)\right|^{2}(v \bullet \zeta), \\
|y-x-(r / 2) \zeta|^{2}= & |y-x|^{2}+\left(r^{2} / 4\right)-r(y-x) \bullet \zeta \\
\geq & |y-x|^{2}+\left(r^{2} / 4\right)-(1 / 4)\left|T_{\natural}(y-x)\right|^{2}(v \bullet \zeta) \geq r^{2} / 4 .
\end{aligned}
$$


Let $c_{0}=\mathscr{L}^{n-1}\left(\mathbf{U}\left(0,1 /\left(1+32^{2}\right)^{1 / 2}\right)\right)$ and we prove that

$$
\mathscr{H}^{n-1}(\mathbf{q}[L]) \geq c_{0} .
$$

We consider the closed convex cone

$$
C=\{(1-t) F(0)+t x: x \in T \cap \mathbf{B}(0,4 r), 0 \leq t<\infty\}
$$

and we notice that

$$
\begin{aligned}
& \text { Dual Nor }(C, F(0))=\operatorname{Tan}(C, F(0))=\{z-F(0): z \in C\}, \\
& \left\{z: z \bullet v \leq 0,\left|T_{\natural}(z)\right| \leq 4 r\right\} \subseteq C .
\end{aligned}
$$

A direct computation shows that

$$
\mathbf{S}^{n-1} \cap \operatorname{Nor}(C, F(0))=\mathbf{S}^{n-1} \cap\left\{\eta: 32 /\left(1+32^{2}\right)^{1 / 2} \leq \eta \bullet v \leq 1\right\},
$$

whence we readily infer that

$$
\mathscr{H}^{n-1}\left(\operatorname{Nor}(C, F(0)) \cap \mathbf{S}^{n-1}\right) \geq c_{0} .
$$

Therefore in order to prove (12) it remains to check that

$$
\operatorname{Nor}(C, F(0)) \cap \mathbf{S}^{n-1} \subseteq \mathbf{q}[L] .
$$

Let $\eta \in \operatorname{Nor}(C, F(0)) \cap \mathbf{S}^{n-1}$. In the case that $(z-F(0)) \bullet \eta \leq 0$ for every $z \in F(A)$, then it is obvious that $(F(0), \eta) \in L$ (notice that $\left.F(0)=(r / 8) v \in C_{r}\right)$. Therefore we assume that $s=\sup \{(z-F(0)) \bullet \eta: z \in F[A]\}>0$. If $z \in F[A]$ and $(z-F(0)) \bullet \eta>0$ then we notice that

$$
\begin{array}{ll}
z \notin C \text { by (13), } & \left|T_{\natural}(z)\right| \leq 4 r \text { by definition of } F, \\
z \bullet v>0 \text { by(14), } & \left|T_{\natural}(z)\right|<r \text { and }\left|T_{\natural}^{\perp}(z)\right|<3 r / 16 \text { by (9), }
\end{array}
$$

that means $z \in C_{r}$. Therefore we select $z_{0} \in F[A]$ such that $\left(z_{0}-F(0)\right) \bullet \eta=s$ and, noting that the maximality of $z_{0}$ implies that

$$
\left(w-z_{0}\right) \bullet \eta \leq 0 \text { for every } w \in F[A],
$$

we conclude that $\left(z_{0}, \eta\right) \in L$.

We notice that $N(F[A])$ satisfies the $m$ dimensional Lusin $(\mathrm{N})$ condition in $F\left[C_{4 r}\right]$ by Lemma 3.4. Therefore, employing Remark 3.3, Theorem 2.2, [17, 4.8] and (11) and noting that $\mathrm{D} F\left(F^{-1}(z)\right)=\mathrm{D} F^{-1}(z)^{-1}$ for $z \in \mathbf{R}^{n}$, we infer at $\mathscr{H}^{n-1}$ a.e. $(z, \eta) \in L$ that

$$
\begin{aligned}
& \operatorname{dim} T_{F[A]}(z, \eta)=m, \quad \mathrm{D} F^{-1}(z)\left[T_{F[A]}(z, \eta)\right]=T_{A}\left(v_{F^{-1}}(z, \eta)\right), \\
& Q_{F[A]}(z, \eta) \geq 0, \\
& Q_{A}\left(v_{F^{-1}}(z, \eta)\right)(\tau, \tau) \geq-(r / 2)^{-1}|\tau|^{2} \text { for } \tau \in T_{A}\left(v_{F^{-1}}(z, \eta)\right), \\
& Q_{F[A]}(z, \eta)=\left|\left(\mathrm{D} F\left(F^{-1}(z)\right)\right)^{*}(\eta)\right| Q_{A}\left(v_{F^{-1}}(z, \eta)\right) \circ \bigodot_{2}\left(\mathrm{D} F^{-1}(z) \mid T_{F[A]}(z, \eta)\right) \\
& \quad-\left(\mathrm{D}^{2} F^{-1}(z) \mid \bigodot_{2} T_{F[A]}(z, \eta)\right) \bullet \mathrm{D} F\left(F^{-1}(z)\right)^{*}(\eta) .
\end{aligned}
$$

In particular, by $[8,2.10 .25]$, the same conclusion holds for $\mathscr{H}^{m}$ a.e. $z \in \mathbf{p}[L]$ and for $\mathscr{H}^{n-m-1}$ a.e. $\eta \in\{\zeta:(z, \zeta) \in L\}$. We combine Theorem 3.7, (16) and the classical inequality relating the arithmetic and the geometric means of a family of non negative numbers (see [15, pp. 29]) to estimate

$$
\mathscr{H}^{n-1}(\mathbf{q}[L]) \leq \int_{\mathbf{S}^{n-1}} \mathscr{H}^{0}\{z:(z, \eta) \in L\} d \mathscr{H}^{n-1} \eta
$$




$$
\begin{aligned}
& =\int_{F[A]} \int_{\{z\} \times\{\eta:(z, \eta) \in L\}} \operatorname{discr} Q_{F[A]} d \mathscr{H}^{n-m-1} d \mathscr{H}^{m} z \\
& \leq m^{-m} \int_{F[A]} \int_{\{z\} \times\{\eta:(z, \eta) \in L\}}\left(\operatorname{trace} Q_{F[A]}\right)^{m} d \mathscr{H}^{n-m-1} d \mathscr{H}^{m} z .
\end{aligned}
$$

We observe that if $z \in \operatorname{Clos} C_{r}, \eta \in \mathbf{S}^{n-1}$ and $S \in \mathbf{G}(n, m)$, then

$$
\begin{aligned}
& \left\|\mathrm{D} F\left(F^{-1}(z)\right)\right\| \leq 3 / 2, \quad\left\|\mathrm{D} F^{-1}(z)\right\| \leq 3 / 2, \\
& \left|\operatorname{trace}\left[\left(\mathrm{D}^{2} F^{-1}(z) \mid \bigodot_{2} S\right) \bullet \mathrm{D} F\left(F^{-1}(z)\right)^{*}(\eta)\right]\right| \leq(3 / 4) m r^{-1} .
\end{aligned}
$$

Therefore, noting (15) and (17), we use Lemma 3.8 to infer that

$$
\begin{aligned}
\operatorname{trace} & {\left[Q_{A}\left(v_{F^{-1}}(z, \eta)\right) \circ \bigodot_{2}\left(\mathrm{D} F^{-1}(z) \mid T_{F[A]}(z, \eta)\right)\right] } \\
\leq & c_{1}\left[\operatorname{trace}\left(Q_{A}\left(v_{F^{-1}}(z, \eta)\right)\right)+r^{-1}\right]
\end{aligned}
$$

whence we readily deduce using (18) and (19) that

$$
\left(\operatorname{trace} Q_{F[A]}(z, \eta)\right)^{m} \leq c_{1}\left(f\left(v_{F^{-1}}(z, \eta)\right)^{m}+r^{-m}\right)
$$

for $\mathscr{H}^{n-1}$ a.e. $(z, \eta) \in L$, where $c_{1}$ is a constant depending only on $m$.

Since $N(F(A))$ is countably $n-1$ rectifiable and $L \subseteq N(F(A))$ one may argue as in $[8,2.10 .26]$ to prove that $D=\left\{z: \mathscr{H}^{n-m-1}\{\zeta:(z, \zeta) \in L\}>0\right\}$ is a Borel subset of $\mathbf{R}^{n}$. Define

$$
M=v_{F^{-1}}[L \mid D],
$$

and notice that it follows from Lemma 2.1 that $M$ is a Borel subset of $N(A)$ with

$$
\mathscr{H}^{n-m-1}\{v:(x, v) \in M\}>0 \text { for } x \in \mathbf{p}[M] .
$$

Moreover $\mathbf{p}[M] \subseteq \operatorname{Clos} C_{(11 / 8) r}$ by (10) and $\boldsymbol{\delta}_{A}(x+(r / 2) u)=r / 2$ whenever $(x, u) \in M$ by (11). Noting that $N(A, z)$ is contained in an $n-m$ dimensional plane whenever $z \in A^{(m)}$ (see Sect. 2.2) and that

$$
\operatorname{Lip}\left(F \mid \operatorname{Clos} C_{(11 / 8) r}\right) \leq \sup _{z \in \operatorname{Clos} C_{(11 / 8) r}}\|\mathrm{D} F(z)\| \leq 27 / 16,
$$

we use Lemma 2.1 to estimate

$$
\begin{aligned}
\left.\int \mathscr{H}^{n-m-1}\{\zeta:(z, \zeta) \in L\}>0\right\} d \mathscr{H}^{m} z \\
\quad \leq \int_{D \cap F(A)} \mathscr{H}^{n-m-1}(N(A, z)) d \mathscr{H}^{m} z \\
\leq \mathscr{H}^{n-m-1}\left(\mathbf{S}^{n-m-1}\right) \mathscr{H}^{m}\left[F\left(A^{(m)} \cap \mathbf{p}(M)\right)\right] \\
\quad \leq \mathscr{H}^{n-m-1}\left(\mathbf{S}^{n-m-1}\right)(27 / 16)^{m} \mathscr{H}^{m}(\mathbf{p}(M)), \\
\int_{F[A]} \int_{\{\zeta:(z, \zeta) \in L\}} f\left(v_{F^{-1}}(z, \eta)\right)^{m} d \mathscr{H}^{n-m-1} \eta d \mathscr{H}^{m} z \\
\leq c_{2} \int_{A} \int_{\{\zeta:(z, \zeta) \in M\}} f(z, \eta)^{m} d \mathscr{H}^{n-m-1} \eta d \mathscr{H}^{m} z,
\end{aligned}
$$

where $c_{2}$ is a constant depending on $m$ and $n$. Therefore,

$$
\mathscr{H}^{n-1}(\mathbf{q}[L]) \leq m^{-m} c_{1} c_{2} \int_{A} \int_{\{\zeta:(z, \zeta) \in M\}} f(z, \eta)^{m} d \mathscr{H}^{n-m-1} \eta d \mathscr{H}^{m} z
$$




$$
+m^{-m} c_{1}(27 / 16)^{m} \mathscr{H}^{n-m-1}\left(\mathbf{S}^{n-m-1}\right) \mathscr{H}^{m}(\mathbf{p}[M]) r^{-m} .
$$

Noting (12), we choose $\delta>0$ in (III) so that

$$
c_{0}-m^{-m} c_{1} c_{2} \delta \geq c_{0} / 2
$$

and we conclude from (20) that

$$
\mathscr{H}^{m}(\mathbf{p}[M]) \geq \sigma r^{m}
$$

with $\sigma=m^{m} c_{1}^{-1}(27 / 16)^{-m} \mathscr{H}^{n-m-1}\left(\mathbf{S}^{n-m-1}\right)^{-1}\left(c_{0} / 2\right)$. Being $\mathscr{H}^{m}(\mathbf{p}[M])>0$ and $\mathscr{H}^{n-m-1}(N(A, a))>0$ for all $a \in \mathbf{p}[M]$ it follows that $\mathbf{p}[M] \subseteq A^{(m)}$.

Proof of Theorem 1.3. Firstly we notice that $C_{4 r}(T, a) \subseteq \mathbf{B}(a, 4 \sqrt{2} r)$ for every $a \in \mathbf{R}^{n}$ and $T \in \mathbf{G}(n, n-1)$. If $\delta$ is given as in Lemma 3.10, with the help of [8, 2.4.11], for $\mathscr{H}^{m}$ a.e. $a \in A \cap \Omega$ we can select $s>0$ and $v \in \mathbf{S}^{n-1}$ such that $\mathbf{B}(a, 4 \sqrt{2} s) \subseteq \Omega$,

$$
r^{-1} \sup \{v \bullet(x-a): x \in \mathbf{B}(a, 4 \sqrt{2} r) \cap A\} \leq 1 / 16,
$$

and

$$
\int_{C_{2 r}(T, a) \cap A} \int_{\{z\} \times N(A, z)} f^{m} d \mathscr{H}^{n-m-1} d \mathscr{H}^{m} z \leq \delta
$$

for every $0<r<s$, where $T \in \mathbf{G}(n, n-1)$ such that $T_{\natural}(v)=0$. It follows from Lemma 3.10 that

$$
\boldsymbol{\Theta}_{*}^{m}\left(\mathscr{H}^{m}\left\llcorner A^{(m)}, a\right)>0 \text { for } \mathscr{H}^{m} \text { a.e. } a \in A \cap \Omega\right. \text {. }
$$

Since $\Theta^{m}\left(\mathscr{H}^{m}\left\llcorner A^{(m)}, a\right)=0\right.$ for $\mathscr{H}^{m}$ a.e. $a \in \Omega \sim A^{(m)}$ by [8, 2.10.19(4)], we infer that

$$
\mathscr{H}^{m}\left(A \cap \Omega \sim A^{(m)}\right)=0 .
$$

The postscript follows from [12, 4.12].

\section{Proof of theorem 1.1}

Here we prove Theorem 1.1. The main point will be to check that the closure ${ }^{6}$ in $\mathbf{R}^{n}$ of the support $S$ of $V$ satisfies the hypothesis of the general criterion for $\mathcal{C}^{2}$ rectifiability in Theorem 1.3. These hypothesis have been already checked for $V$ in several different papers, so we just need to collect them here.

(1) $\mathscr{H}^{m}(S \cap K)<\infty$ for every compact set $K \subseteq \Omega$. This follows combining the uppersemicontinuity of the density function $\Theta^{m}(\|V\|, \cdot)$, see $[1,8.6]$, with the fact that $\|V\|=$ $\mathscr{H}^{m}\left\llcorner\Theta^{m}(\|V\|, \cdot)\right.$. In fact, we obtain the stronger conclusion $\mathscr{H}^{m}\left\llcorner S \leq \theta^{-1}\|V\|\right.$.

(2) $N(\operatorname{Clos} S)$ satisfies the $m$ dimensional Lusin $(N)$ condition and

$$
\text { trace } Q_{\mathrm{Clos} S}(a, u) \leq h \text { for } \mathscr{H}^{n-1} \text { a.e. }(a, u) \in N(\operatorname{Clos} S) \mid \Omega \text {. }
$$

Noting [23, 2.8], this is a special case of [18, 3.7].

(3) For $\|V\|$ a.e. $a \in \Omega$ there exists an $m$ dimensional plane $T$ such that

$$
\lim _{r \rightarrow 0} r^{-1} \sup \left\{\delta_{T}(x-a): x \in \mathbf{B}(a, r) \cap S\right\}=0 .
$$

This follows from $[21,17.11]$.

6 We take the closure in $\mathbf{R}^{n}$ because Theorem 1.3 has been formulated for closed subsets in $\mathbf{R}^{n}$. 
Acknowledgements The results of this paper were proved when the author was a Phd Student under the supervision of Prof. Ulrich Menne at the Max Planck Institute for Gravitational Physics. The author is grateful to his Phd advisor for his constant and supportive guidance throughout the preparation of this work.

Funding Open Access funding enabled and organized by Projekt DEAL.

Open Access This article is licensed under a Creative Commons Attribution 4.0 International License, which permits use, sharing, adaptation, distribution and reproduction in any medium or format, as long as you give appropriate credit to the original author(s) and the source, provide a link to the Creative Commons licence, and indicate if changes were made. The images or other third party material in this article are included in the article's Creative Commons licence, unless indicated otherwise in a credit line to the material. If material is not included in the article's Creative Commons licence and your intended use is not permitted by statutory regulation or exceeds the permitted use, you will need to obtain permission directly from the copyright holder. To view a copy of this licence, visit http://creativecommons.org/licenses/by/4.0/.

\section{References}

1. Allard, W.K.: On the first variation of a varifold. Ann. Math. 2(95), 417-491 (1972)

2. Anzellotti, G., Serapioni, R.: $\mathscr{C}^{k}$-rectifiable sets. J. Reine Angew. Math. 453, 1-20 (1994)

3. Brakke, K.A.: The Motion of a Surface by Its Mean Curvature. Mathematical Notes, vol. 20. Princeton University Press, Princeton (1978)

4. Caffarelli, L.A., Cabré, X.: Fully Nonlinear Elliptic Equations. American Mathematical Society Colloquium Publications, vol. 43. American Mathematical Society, Providence (1995)

5. De Rosa, A., Kolasiński, S., Santilli, M.: Uniqueness of critical points of the anisotropic isoperimetric problem for finite perimeter sets. Arch. Ration. Mech. Anal. 238(3), 1157-1198 (2020)

6. Duggan, J.P.: Regularity theorems for varifolds with mean curvature. Indiana Univ. Math. J. 35(1), 117144 (1986)

7. Federer, H.: Curvature measures. Trans. Am. Math. Soc. 93, 418-491 (1959)

8. Federer, H.: Geometric Measure Theory. Die Grundlehren der mathematischen Wissenschaften, Band, vol. 153. Springer, New York (1969)

9. Menne, U.: Decay estimates for the quadratic tilt-excess of integral varifolds. Arch. Ration. Mech. Anal. 204(1), 1-83 (2012)

10. Menne, U.: Second order rectifiability of integral varifolds of locally bounded first variation. J. Geom. Anal. 23(2), 709-763 (2013)

11. Menne, U.: Weakly differentiable functions on varifolds. Indiana Univ. Math. J. 65(3), 977-1088 (2016)

12. Menne, U., Santilli, M.: A geometric second-order-rectifiable stratification for closed subsets of Euclidean space. Ann. Sc. Norm. Super. Pisa Cl. Sci. (5) 19(3), 1185-1198 (2019)

13. Martio, O., Ziemer, W.P.: Lusin's condition (N) and mappings with nonnegative Jacobians. Mich. Math. J. 39(3), 495-508 (1992)

14. Nadirashvili, N.S.: Certain differential properties of solutions of elliptic equations with measurable coefficients. Izv. Akad. Nauk SSSR Ser. Mat. 49(6), 1329-1335, 1344 (1985)

15. Rockafellar, R.T.: Convex Analysis. Princeton Mathematical Series, No. 28. Princeton University Press, Princeton (1970)

16. Santilli, M.: Rectifiability and approximate differentiability of higher order for sets. Indiana Univ. Math. J. 68, 1013-1046 (2019)

17. Santilli, M.: Fine properties of the curvature of arbitrary closed sets. Ann. Mat. Pura Appl. (4) 199(4), $1431-1456(2020)$

18. Santilli, M.: Normal bundle and Almgren's geometric inequality for singular varieties of bounded mean curvature. Bull. Math. Sci. 10(1), 2050008, 24, (2020)

19. Schätzle, R.: Quadratic tilt-excess decay and strong maximum principle for varifolds. Ann. Sc. Norm. Super. Pisa Cl. Sci. (5) 3(1), 171-231 (2004)

20. Schätzle, R.: Lower semicontinuity of the Willmore functional for currents. J. Differ. Geom. 81(2), 437456 (2009)

21. Simon, L.: Lectures on geometric measure theory. In: Proceedings of the Centre for Mathematical Analysis, vol. 3. Australian National University. Australian National University, Centre for Mathematical Analysis, Canberra (1983)

22. Trudinger, N.S.: On the twice differentiability of viscosity solutions of nonlinear elliptic equations. Bull. Aust. Math. Soc. 39(3), 443-447 (1989) 
23. White, B.: Controlling area blow-up in minimal or bounded mean curvature varieties. J. Differ. Geom. 102(3), 501-535 (2016)

Publisher's Note Springer Nature remains neutral with regard to jurisdictional claims in published maps and institutional affiliations. 